\title{
EXPLORATORY ANALYSIS TO ADDRESS DEEP UNCERTAINTY - USING CALIBRATABLE SYSTEM MODELS FOR EXPLORATORY SIMULATION OF COMPLEX MISSIONS
}

\author{
Andreas Tolk \\ Khuong Dinh \\ The MITRE Corporation \\ 903 Enterprise Parkway \#200 \\ Hampton, VA 23666, United States \\ \{atolk, kdinh\}@mitre.org
}

\author{
Kevin Comer \\ Steve Scott \\ The MITRE Corporation \\ 7515 Colshire Drive \\ McLean, VA 22102, United States \\ $\{$ ktcomer, slscott\}@mitre.org
}

\begin{abstract}
Traditional optimization methods and sometimes even the underlaying concept of experimentation design for optimization problems are not always successful in complex solution spaces. This is not only driven by the complexity itself, but also by the deep uncertainty in estimating the model parameters. Applying exploratory analysis to better understand the solution space has been recognized as a promising approach for several years, but with the new generation of computers the community now has the means to practically apply it as well. We define the terms, derive a conceptual model, provide an application case from the defense domain, and show the possibility to generalize the results, namely that parameter sweeps are possible and lead to deeper insights of the solution space, allowing for stable and robust solutions that often are more important in practice than optimized point solutions.
\end{abstract}

Keywords: complexity, deep uncertainty, exploratory analysis.

\section{INTRODUCTION}

The topic of complexity is not new to Modeling and Simulation (M\&S) experts. Different viewpoints, methods, and research results have been published and discussed since the early 1960s under the topic of system science or cybernetics. The applied M\&S community has been interested in the complexity science domain for more than a decade as well, providing extensive work in various complexity science approaches, but predominantly applying agent-based modeling methods. The Guided Tour to Complexity by Mitchell (2009) gives a good overview of such engagements and applied methods. Among the notable contributions of M\&S are the contributions of Axtell and Epstein in various fields, such as their work on growing artificial societies (Epstein and Axtell 1996) and generative social science (Epstein 2006). More recent publications on M\&S and complexity show connections to systems engineering (Diallo et al. 2018) and cyber-physical systems (Mittal and Tolk 2019).

Complexity and deep uncertainty require us to rethink how to use M\&S for decision support in research domains like discussed in this paper. The traditional approach for simulation-based optimization is captured in nearly every simulation textbook: formulate the problem, collect data, construct the computer software, design the experiment, execute the simulation, analyze the data, and finally present the results. With the currently available computer power, the models can reach even higher levels of resolution and increase 
their fidelity. However, if the solution space is complex and the research question deeply uncertain, higher resolution and more fidelity of a problem that is ill specified or may even be wicked and is of no value. The idea of exploratory analysis and the search for strategies that are flexible, adaptive, and robust arose in defense planning (Davis 1994) with multi-scenario analysis and what came to be capabilities-based planning arose in parallel in research to understand implications of complexity theory for analysis (Bankes 1993).

To deal with these challenges and motivate our proposed solution, this paper is organized as follows. Section 1 provides an introduction to the problem domain, and introduces the concepts of complexity, deep uncertainty, and exploratory analysis. Section 2 provides more detailed exposition of these topics. Section 3 introduces conceptual frameworks for conceptual modeling under uncertainty, including the ModelView-Controller (MVC) and Mission-Means-Framework (MMF). Section 4 discusses an application of these concepts to a military simulation domain, examining how these approaches are relevant for exploring the US Army Multi-domain Operations (MDO) problem space as an example. Section 5 discusses results, extends these ideas to other non-military domains such as economics, and considers future work including the study of emergence in complex systems, the use of governance tools, and the development of common conceptual models based on standardized formalisms.

\section{CONCEPTS AND DEFINITIONS}

Within the title, we are introducing three concepts that require definitions, as they are used with different meaning in various contexts. These concepts are complexity, deep uncertainty, and exploratory analysis. All three concepts literally fill volumes by their own right, so we will provide a broader view for each, and then use this broader view to specify in more detail which facets we are addressing with our research.

\subsection{Complexity}

In the context of this research, we were mainly interested in complex solution spaces. These solution spaces are characterized by the high interconnectedness of its components, with many non-linear connections, and characteristics attributes of the system being exposed by these interconnections, and not only by the components. As a result, only small variations can result in significant changes in the outcome, the same components in slight variations can build different relations, which results in a different behavior, and as behavior may emerge out of new relations bearing characteristic attributes, the application of reduction doesn't work, as dividing the system into its subcomponents changes the system behavior. Tolk et al. (2020) provide examples from the defense and the business domain, showing the conceptual similarity of their challenges on the abstract level of system representation. While complexity is not a new phenomenon, the increase of systems capabilities did lead to an increase in complexity. This can be observed in many domains, such as in social systems, where technology and modern travel options today allow for worldwide social net-structures, business application, where worldwide consortia and rapidly changing contracts are the norm, or in the defense sector, where new weapon systems allows to overlap effects beyond the usual borders. As a result, the recommendations derived by the application of many traditional methods in a complex solution space may not lead to the desired outcome. A robust acceptable solution may be better than an optimized spot solution, as will be discussed in the section of exploratory analysis in more detail. Methods that assume quasilinear behavior and strong correlation in their interpretation are no longer sufficient. Reductionism may also lead to misleading results (Gallagher and Appenzeller 1999), as a broader system understanding is needed.

\subsection{Deep Uncertainty}

Lempert et al. (2003) define deep uncertainty as conditions "where analysts do not know, or the parties to a decision cannot agree on, (1) the appropriate conceptual models that describe the relationships among the key driving forces that will shape the long-term future, (2) the probability distributions used to represent uncertainty about key variables and parameters in the mathematical representations of these conceptual 
models, and/or (3) how to value the desirability of alternative outcomes." In other words, deep uncertainty is systemic in the research. Parameters of systems may be unknown, behaviors and roles are unclear, objectives to be reached are still in question, etc.

The reasons for deep uncertainty can be multifold. The research question can cope with future systems for which we can only assume their capabilities. The research may analyze the business behavior of a competitor, which is not known, or a weapon system of the opposing force, with highly classified and hard to observe technical parameters.

Traditional operations methods can help to understand and cope with facets, but they do not address the kernel of the problem: the inability to agree on the underlying problem itself. Nonetheless, they can and should contribute to understand facets of the challenge. Velasquez and Hester (2013) conducted a literature review and analysis of multi-criteria methods. Slantchev (2012) defines preferences and utilities to support decision making, including those to be made under uncertainty. A game theoretic variant for multi-issue closed negotiations addressing multi-time as well as multi-lateral negotiation strategies is described by Matsune and Fujita (2017), who developed not only the concept, but demonstrated them in an agent-based simulation environment. It should be pointed out that all traditional methods are still valuable, they just have to be used in orchestration, as already apparent from the complexity discussion.

\subsection{Exploratory Analysis}

Exploratory analysis accepts deep uncertainty as a premise. As a result of this deep uncertainty, all parameters of a model can and should be modified. While traditional approaches focus exclusively on the free parameters (understood as those independent variables that are being tested directly), exploratory analysis modifies the bound parameters as well, or those parameters assumed to be fixed or constant. Even the target parameters, those that provide the output metrics of the model, may be modified, as well as the functions used to capture policies and procedures of the research domain. In other words, a broad parameter sweep allows to get an understanding of the solution space instead on focusing on a high-resolution point solution, which may turn out to be in an irrelevant section of the solution space. Related strands of research sprung up in Europe, as in work on technology and social forecasting by Kwakkel and Pruyt (2013). An international society for decision-making under deep uncertainty compiled relevant methods in (Marchau et al. 2019). While the theoretic value is known since the seminal work by Bankes (1993), the recent advances on computational capabilities and accessibility to such computational means do allow for practical applications, even for large parameter spaces.

\section{CONCEPTUAL MODELING FOR ANALYSIS UNDER COMPLEXITY}

The traditional approach for using simulation in the analysis of systems involves high resolution and fidelity models, specifically validated to empirical data on a well-known system. This current approach does not address two separate needs for the purposes of capturing the underlying complexity of the system. The inability to capture the inherent architectural complexity and plausibility of emergent behavior limits one's ability to perform exploratory analysis on the system, given that the simulation's high fidelity is only validated to within very specific measures. Similarly, this limits the generalizability of the simulation model towards other domains, thus hindering the sharing of information and reuse of solutions within other complex environments, which have been shown to have common issues that can be addressed using similar methods (Chen and Crilly 2016).

To address these perceived gaps in the traditional approach, this paper seeks to describe a methodology for producing a domain-agnostic conceptual model that captures the concepts, their relations, processes, and functions of a system of interest within its context. This allows the system to be conceptualized in its operational context, and so the model can be used to express the various observations of complexity and

emergence. To facilitate practical use and access to the concepts, various domain-specific views are planned 
for implementation so that domain experts can instantiate domain specific applications of the common concepts. One such domain-specific implementation is discussed in Section 4.

The conceptual model framework for the purposes of exploratory analysis in complex systems extends from two existing paradigms - the model-view-controller paradigm centered around the relationship between the user and the analysis, and the mission and means framework for the evaluation of solutions.

\subsection{Model-View-Controller Paradigm}

The model-view-controller (MVC) paradigm was developed to allow multiple graphical user interfaces that all interact with the same model. It is a software development architecture pattern that attempts to abstract the user, and the users' need for data presentation, from the underlying model. The idea is to separate the model from the user, who can drive different application presentations or views. The user would use a controller interface to request data or a presentation, which would be answered by a model, then sent back to the user as a view for consumption. Figure 1 shows these principles by drawing a box around the model, view, and controller while leaving the user outside the software application space.

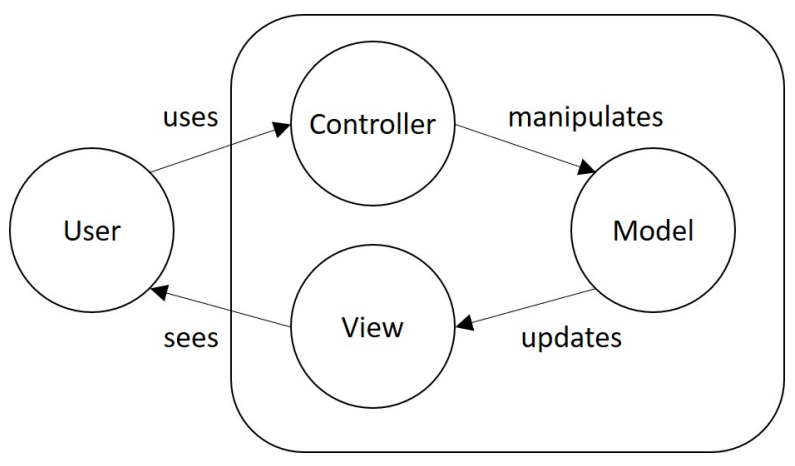

Figure 1: Model-View-Controller Paradigm.

By extending the MVC paradigm to include domain-specific modeling elements in the views and controllers, such as objects and their corresponding functions, the conceptual model can be generalized to capture operational complexity in a variety of domains and environments. The systems of interest are defined in the views and the controllers, as are the domain specific attributes, thus providing a familiar interface for display and manipulation to the subject matter expert. Importantly, the views and controllers are connected to common concepts, and an emerging effect, based on components of the conceptual model, will be shown in the domain-specific interpretations of the various viewers. This approach allows a domainagnostic model that focuses on the underlying concepts identified in the conceptual model while at the same time providing domain experts with a representation of these concepts as instantiations in their domain.

\subsection{Missions and Means Framework}

Another inspiration for our research was the missions and means framework (MMF), developed for the evaluation of capability-based procurement ideas (Sheehan 2004). It distinguishes between the capabilities required to conduct a military mission and the means provided by the acquisition portfolio of interest. The levels represented in this framework include not only the capabilities and functions of blue and red operations, but also the operational context and environment which applies to both forces. In addition to the hierarchical structure of this framework, there is the multi-scale decomposability of the framework, allowing for the mission elements to be considered and analyzed separately from the means elements. This conceptual separation of what needs to be accomplished (mission) and how these objectives are accomplished (means) guided the work on our conceptual model.

Although the MMF was designed for the defense domain, note that this language is not specific to military operations, but rather applies equally to non-military and commercial activities (i.e., missions). The domain-agnostic vocabulary allowing for the decomposability between means and missions allow for the 
robust analysis of alternatives, identifying means geared towards the successful end of tasks, objectives, and missions, given the complex environment. The hierarchical multi-scale nature of this framework allows for the operational complexity to not only be present in the conceptual model, but to be the driving force behind identifying key solution spaces for further analysis.

\subsection{Conceptual Model of Operational Complexity}

The two frameworks discussed above address actualized, critical problems in systems analysis, but they are not without their shortcomings, especially in terms of addressing deep uncertainty and exploratory analysis. The MMF delineates in concrete terms the interactions of the domains in consideration - thus emphasizing complexity - but does not seek to examine the uncertainty of the bound parameters that those interactions rely on. In contrast, the MVC framework is abstract enough to model deep uncertainty - the testing of bound parameters - but it is too abstract to permit much structured exploratory analysis. The goal of the conceptual model discussed in this paper is to address this balance between abstraction and functionality in understanding operational complexity.

The conceptual model, as implemented in the core model as a high-level view of the conceptual elements and their interplay, can be seen depicted in Figure 2. The upper half of the model-Mission and Capabilities/Tasks - is the operational domain. The lower half of the model—Functionality, Controls, and Systems - is the conceptual domain.

At the highest level of the model shown in Figure 2 is the operational mission, which sets the overall objective and rationale for undertaking actions. This can be a military mission, but also a strategic business plan or a set of healthcare policy goals. The mission is decomposed into tasks predicated on operational functionality, which is referred to as a required capability. A capability is agnostic as to which system will use which function to provide the capability. A capability is mission-oriented, while a functionality is systems-oriented. In simple terms, systems (with their claimed functionalities) when put together in a context, may offer a capability that is usable by a mission. Conversely, a mission may define or require a capability and assembles a set of systems that provide redundant functionality.

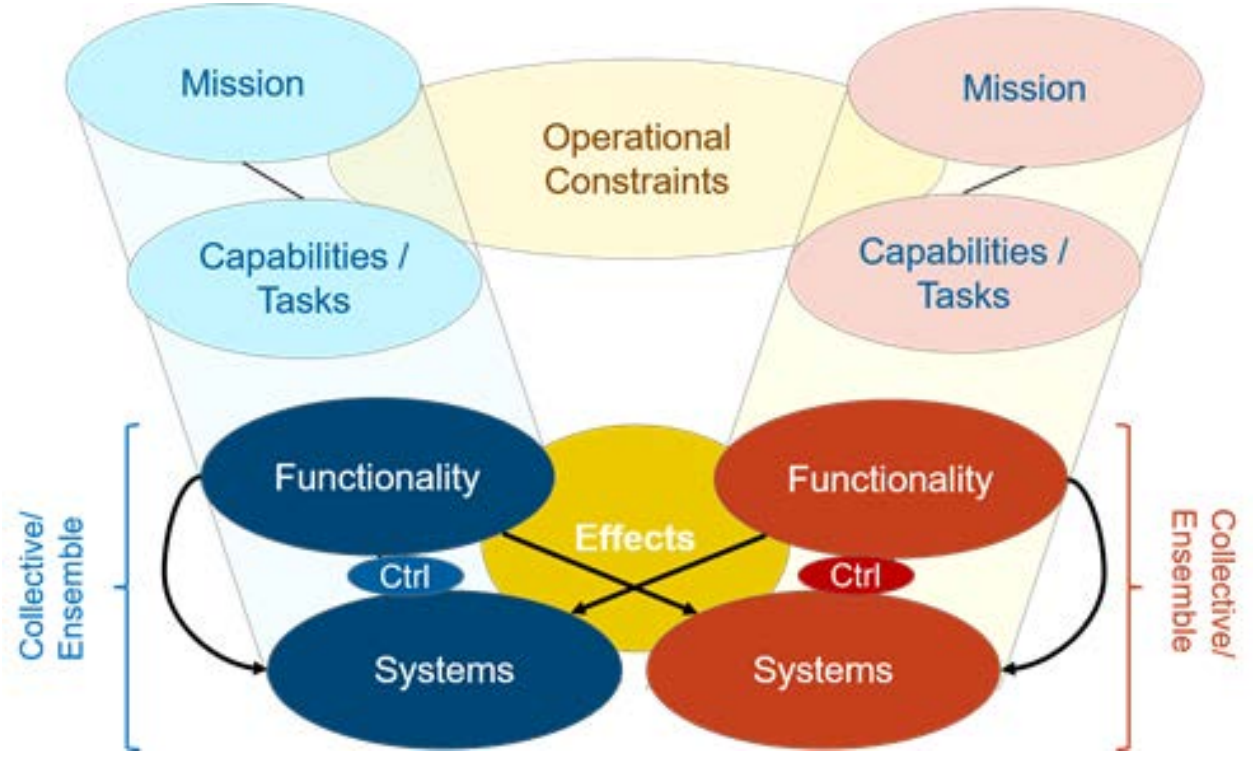

Figure 2. Conceptual Model high-level view.

The conceptual model is predicated on a set of building blocks, which are the model entities. Each entity has a set of attributes that define the entity. Entities can also host a set of functions, which manipulate the attributes of entities. These entities are instantiated as objects in a situated virtual environment, that may expose the same attributes as the entities. Functions are invoked to provide services, which are defined as 
the change of attributes. The collective execution of these functions creates effects. As the entities are the building blocks, the chosen level of abstraction defines their interpretation as real-world objects, so in the military domain, an entity can be a tank, or a tank platoon, a tank company, and so forth.

While an object provides functions through manipulation of attributes, it does not offer control. An entity that provides additional functions that control various attributes is addressed as a system. To be more specific, if an object resides within a command and control structure, it is as a system. This system may reside either in blue side or red side. The easiest form of command and control is to limit or enforce the use of certain functions of the system via configurable controls. Depending on the level of system autonomy, the decision of which systems to use or not to use (which implies what configuration to use for controls) can be set from inside the system or needs to be set from the outside of the system. As the control set is part of the system, a function is needed to initiate and modify the control settings.

Systems may operate in a group. This group that is deliberately designed, such as a brigade that combines its weapon systems to allow for combined arms operations, is defined as an ensemble. An ensemble is also a system (a system-of-systems, to be more precise) that has mission goals. Alternatively, if such a group emerges from a situation in the environment, this group is called a collective. However, both categories of groups will not replace the comprised systems, because they are only a structure that identifies the composition. The collectives are run-time emergent ensembles and do inherit the ensembles' mission. However, it cannot be determined a priori what the operational control structure comes to be in each context for these collectives. In summary, there is a dependency between the mission, its task, the required capabilities, and the possible functions. As stated, functionality is provided by systems - whether they are logical or physical - and so there is a coupling between the functionality and the system or systems that provide it. This functionality gives the structure needed for directing exploratory analysis, as missions reliant on their lower-order elements impact the output of the model. Additionally, there is a control element within the operational environment, which is part of the Command and Control function. These command and control functions of the mission are by themselves functions that may be aligned with one of the constituent systems. These command and control functions of the mission, as well as their parameterization, provide the ability for testing deep uncertainty, by testing previously held assumptions and addressing the impact of these assumptions being proven untrue.

\section{A DEFENSE EXAMPLE}

The United States Army Futures Command (AFC) is currently evaluating concepts for Multi-Domain Operations (MDO) to conduct continuous and rapid integration of multi-domain (Air, Land, Sea, Cyber, and Space) capabilities to gain cross-domain overmatch at decisive spaces. Decisive spaces are locations in time and space (physical, virtual, and cognitive) where the full optimization of the employment of crossdomain capabilities generates a marked advantage over an enemy and greatly influences the outcome of an operation (TRADOC Pamphlet TP525-3-1 2018). However, there are many uncertainties on the capabilities of the own and the opposing forces, applied tactics, technics, and procedures, control structures, and many more parameters, resulting in deep uncertainty. The use of exploratory analysis to understand the "unknown-unknowns" is critical for this mission as systems and concepts are a mix of current and future systems of systems and the interoperability and Command and Control structure are not clearly defined nor understood.

For the MDO experimentation example, the M\&S and Analysis Team focused on the Joint Suppression of the Enemy Air Defense (JSEAD) scenario to explore the deep uncertainty within the Command and Control (C2) structure and the effects of Intelligence, Surveillance, and Reconnaissance (ISR) across multiple domains to achieve tactical advantages over the adversary. Multi-domain functions and systems included in the experiment consisted of Space, Air, Land systems such as satellites that served as the sensing and ISR functionalities, Fighter Aircrafts and Bombers for sensing and striking, and Long Range Precision Fires (LRPF) and Air Defense systems such as the Patriot for force protection. This problem is complex, as the systems under command of various domains overlap in the area of their possible effects and can be 
combined into collectives as well as ensembles. For example, an opponent can be engaged by air crafts, by long range artillery, or by naval fire, which can be guided by land-, air-, or space based ISR systems. As some of these systems only exist as ideas, there is deep uncertainty about their parameters. The advantage of this constraint is that it also opens the opportunity for better trade spaces, allowing to ask research question such as "Should we procure more swarm-like ISR systems with less reliable capabilities? Or is it better to procure a smaller portfolio of highly reliable systems?" Another set of question could be "What degree of reduction in my command and control capabilities can I tolerate before my portfolio can no longer fulfil its mission?"

In order to address these questions, all parameters were designed as open and modifiable, including weapon system parameters, which allowed to sweep and scan through the solution space as required by the theoretic framework described in the last section. To show that this is feasible and has practical relevance, the team conducted parameter sweeps and executed multiple factors/levels analysis to cover the significant part of the solution space by performing Monte-Carlo simulation using the Behavior Space functionality within NetLogo, shown in Figure 3. The resulting data were stored as comma separated values and analyzed using the SAS JMP Statistical Software to support our objective of exploratory analysis. Isolating each factor (such as command and control, ISR, and Long Range Fires) to determine effects and outcome of battle based on blue/red configuration Multivariate analysis allowed for the exploration of convergence (layered options of multiple domains) that contributed to the most impact (win).

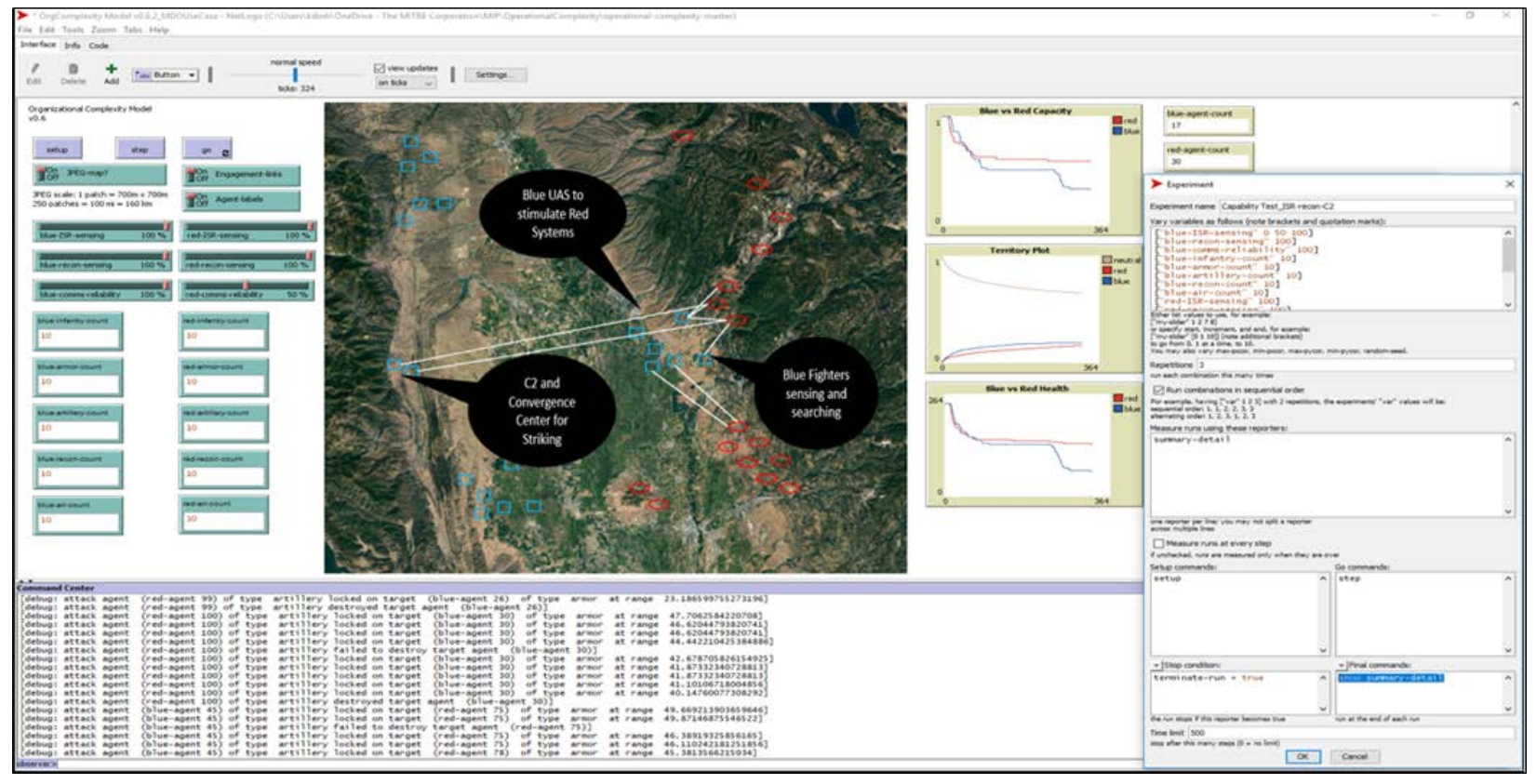

Figure 3: Exploration of the Solution Space.

Based on the data collected from the JSEAD example, as shown in Figure 4, we were able to identify parameters that contributed to a win/lost with a mix of ISR, C2, and LR Fires. Using the Wilcoxon hypothesis testing method, the team discovered that counter to our null hypothesis that the greater the numbers of capacity, the more victory. On the contrary, capacity played a lesser role than that of the $\mathrm{C} 2$ orchestration of convergence and between the ISR and LR Fires capabilities to achieve the victory state, thus requiring further evaluation of a Joint All Domain Command and Control. 


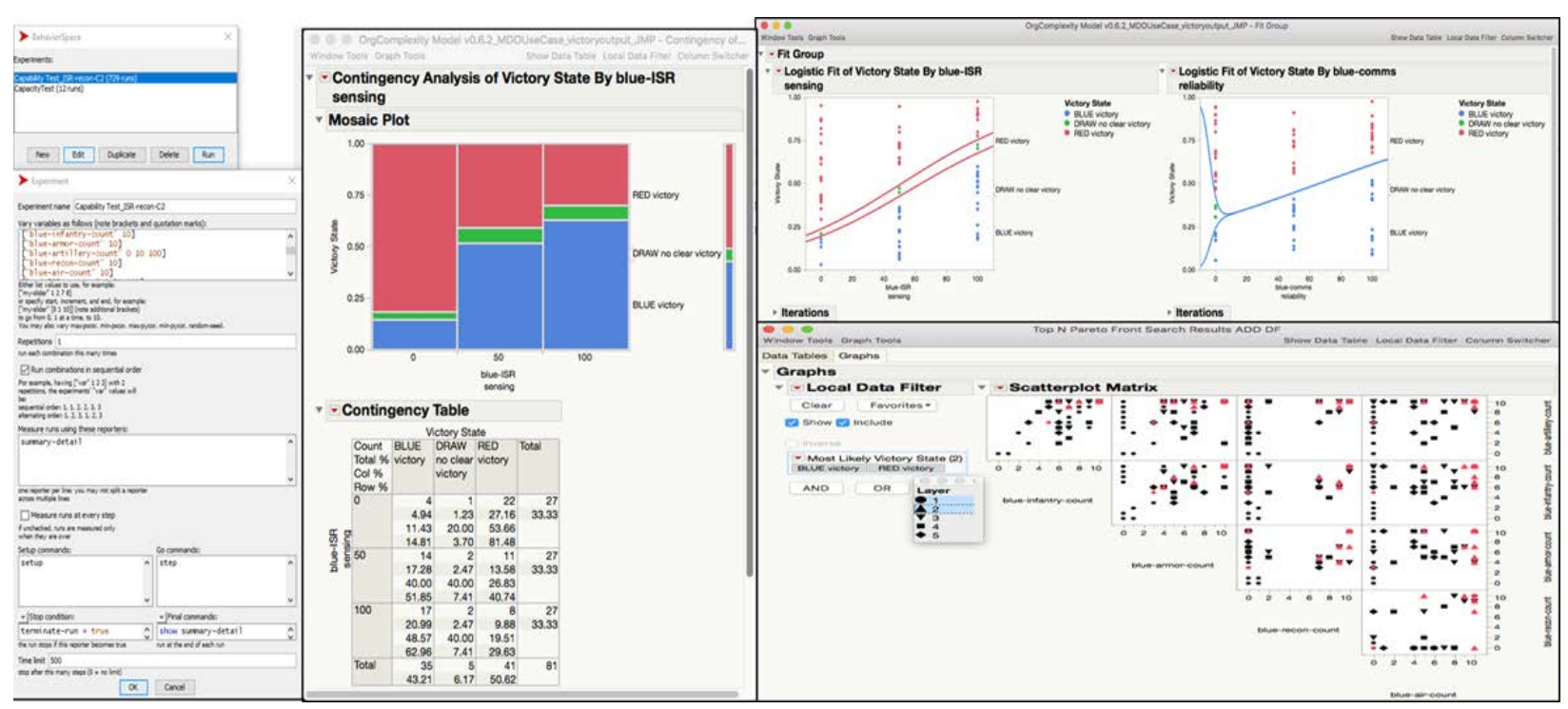

Figure 4: MDO Use Case Exploratory Analysis.

The following example shows one of the counterintuitive effects typical in such complex environments. In the JSEAD analysis, a fifth-generation platform ("Blue-Air" in the model) is well-integrated into a mission and gets an improved standoff weapon capability - one of the varied parameters of the model - allowing for target engagements at further distances. The result is an increase to platform survivability, as the system doesn't have to be in dangerous, highly contested areas as long as before. As a result, less attacks on "BlueAir" are observed, as it was intended. However, reducing the need to penetrate contested areas has an unintended, counterintuitive effect as well: more overall engagements were lost when the improved standoff weapon was used. The research showed that, because the aircraft no longer penetrates hostile airspace, it also no longer collects the intelligence and surveillance information to feed back to the other systems in the portfolio as it did during the penetration mission. As a result, these systems could no longer engage in the battle for the lack of good targeting data, and hence the overall mission value is decreased. The commander would see a decrease in situational awareness through a loss of valuable ISR data because no other system is capable of surveilling as deeply into the contested areas.

Overall, the operational capability of mission packages (or perhaps the operational enterprise) degrades, due to this system-level optimization. Adding additional alternative ISR within the parameter sweep showed that areas in the solution space exist where the improved standoff capability indeed increase the number of wins, but only if additional ISR systems were engaged as well.

\section{DISCUSSION}

Complexity requires quick reaction to changes. This new environment is the result of agile and adaptive opponents who take full advantage of new technical developments and operating concepts. Our own new operational environment necessitates an equally agile option, which facilitates rapid adjustments as demanded by new circumstances. This is true for defense, health, business, and many other domains.

\subsection{Research Results}

The research provided an example of defense modeling and simulation in the Multiple Domain Operations (MDO) scenario. The MDO scenario is tailored for an environment such as the US Army United States Army Futures Command (AFC) which is currently evaluating concepts for MDO missions. This scenario includes the use of space and stratospheric assets (satellites and high altitude airships), atmospheric assets (fighter, electronic warfare, bomber, and unmanned aircraft), and ground based assets (high mobility artillery rocket, extended range cannon, mobile short range air defense, and medium range air defense such 
as PATRIOT). Due to time and budget constraints, only a subset of this mission could be implemented and tested, with focus on coordinated activities between sensors and various shooter options, mainly long-range artillery systems and fighter aircrafts. Using the Monte-Carlo approach, the simulation was run multiple times using various parameter settings in order to characterize the interactions of interacting systems in the context of the MDO scenarios. Data generated by each simulation run was collected and analyzed, resulting in an improved understanding of how variations in values for key parameters affect expected outcomes. In particular, the ability to quickly identify associations (or the lack thereof) between parameter settings and Red/Blue victory conditions is valuable to stakeholders and decision makers.

\subsection{Generalizability of these Results}

While the modeling and simulation activities in this research have been focused on military domains, the principles being demonstrated are by no means limited to defense settings. It is possible to use these techniques to model, for example, the strategic interactions among nation-state trading partners engaged in tariff disputes, firms competing in a market space, or policy makers evaluating competing options for the provision of public goods. The approach requires the definition of two or more parties with conflicting interests, the definition of their associated attributes and capabilities, and the definition of a simulated time and space for them to interact.

\subsection{Future Research}

One of the main insights of this research, which has been supported by several parallel activities of other researchers as well, is the lack of support to detect, recognize, manage, and govern emergence in complex environments (Diallo et al. 2018). The International Council on Systems Engineering (INCOSE) recommends the use of several methods and tools of the M\&S community in their primer for understanding complexity (Sheard et al. 2015). Simulation please a pivotal role, and our research leads to three recommendations for future research topics.

- Experience complexity and emergence of highly non-linear, interconnected systems by providing complex, highly immersive environments. The role of $M \& S$ is here education of decision managers to be able to think in such interconnected systems, where the linear solving of one challenge may result in unforeseen consequences in other domains, such as it is the case in global business, healthcare systems, and international defense challenges.

- Provide governance tools that help to detect, understand, and manage effects, and that help to identify areas where robust solutions are possible as well as areas that should be avoided, because the system behavior is highly counterintuitive and can even be chaotic.

- Develop common conceptual models based on common formalisms that allow for the transfer of research results and insights between different application domains, e.g., using insights from international business to be applied in health care, etc.

The research also motivated the need for operational agility, which is the ability of a local decision maker to utilize all available resources, as these resources can quickly and easily be assembled to provide the necessary capability (Tolk et al. 2020). These results support the predictions made within the NATO Netenabled Command and Control Maturity Model (N2C2M2) by Alberts et al. (2010).

\section{CONCLUSION}

Simulation engineers need to follow a similar paradigm shift as systems engineers needed to follow with the insight on the existence and nature of wicked problems, first discussed by Rittel and Weber (1973): In the era of complexity, we may have to replace control with governance, and optimization with robustness. Instead of searching for high-resolution point-solutions based on subject matter expertise, using exploratory analysis to better understand the solution space is an essential contributions. The intensive sweep of the 
parameter space allows for solutions to emerge that are often not foreseen by experts. If many non-linear, overlapping effects are combine, human experts often are not able to cope with them. As these are future developments introducing innovative systems, behaviors, and procedures, human experts can no longer rely on their expertise, and the use of analog reasoning may mislead them. Like in chaotic systems, stable and non-stable region may be close to each other. The research presented in this paper shows a possibility to identify these characteristics of the solution space, discover unexpected behavior and emerging effects, and replace best guess with well documented, reproducible results.

As the recommended solutions of our research could be transferred to be used in the work force of analysist supporting our sponsors, the recommendations presented in this paper are perceived to be feasible, practical, and make first contributions into the direction of using modeling and simulation means to experience complexity, provide governance, and develop common concepts. Hopefully, it will lead to increased cooperation between complexity researchers, system engineers, and M\&S experts

\section{DISCLAIMER AND ACKNOWLEDGMENTS}

The authors like to thank the research team and everyone who contributed to the ideas captured in this paper, in particular Kris Rosfjord, Saurabh Mittal, Thom DeCarlo, and Howard Lazoff. The work presented in this paper was funded by the MITRE innovation program. The views, opinions, and/or findings contained in this paper are those of The MITRE Corporation and should not be construed as an official government position, or decision, unless designated by other documents. Approved for Public Release; Distribution Unlimited. Case Number 19-01906-15.

\section{REFERENCES}

Alberts, D.S., R. K. Huber, \& J. Moffat. 2010. NATO NEC C2 maturity model. Command Control Research Program, Washington, DC, 2010.

Bankes, S. C. 1993. Exploratory modeling for policy analysis. Operations research 41(3): 435-449.

Chen, C.-C., and N. Crilly. 2016. "Describing Complex Design Practices with a Cross-Domain Framework: Learning from Synthetic Biology and Swarm Robotics." Research in Engineering Design, 27 (3): 291305.

Davis, P. K. 1994. New Challenges in Defense Planning: Rethinking How Much is Enough. RAND Technical Report, Santa Monica, CA.

Diallo, S. Y., S. Mittal, \& A. Tolk. 2018. Emergent Behavior in Complex Systems Engineering: A Modeling and Simulation Approach. John Wiley \& Sons, Inc.

Epstein, J. M. \& R. Axtell. 1996. Growing artificial societies: social science from the bottom up. Brookings Institution Press.

Epstein, J. M. 2006. Generative social science: Studies in agent-based computational modeling. Princeton University Press

Gallagher, R., \& T. Appenzeller. 1999. Beyond reductionism. Science 284(5411): 79-80.

Kwakkel, J. H. \& E. Pruyt. 2013. Exploratory Modeling and Analysis, an approach for model-based foresight under deep uncertainty. Technological Forecasting and Social Change 80(3): 419-431.

Lempert, R. J., S. W. Popper, \& S. C. Bankes. 2003. Shaping the Next One Hundred Years: New Methods for Quantitative, Long-Term Policy Analysis, RAND Technical Report MR-1626, Santa Monica, CA, USA.

Marchau, V. A. W. J., W. E. Walker, P. J. T. Bloemen, \& S. W. Popper (eds.). 2019. Decision Making Under Deep Uncertainty: From Theory to Practice. Springer, Cham, Switzerland. 
Matsune, T. \& K. Fujita. 2017. Weighting estimation methods for opponents' utility functions using boosting in multi-time negotiations. In Agents (ICA), 2017 IEEE International Conference on (pp. 2732). IEEE Press.

Mitchell, M. 2009. Complexity: A guided tour. Oxford University Press

Mittal, S. \& A. Tolk. 2019. Complexity Challenges in Cyber Physical Systems: Using Modeling and Simulation $(M \& S)$ to Support Intelligence, Adaptation and Autonomy. John Wiley \& Sons, Inc.

Rittel, H. \& M. Weber. 1973. Dilemmas in a general theory of planning. Policy Sciences 4:155-169.

Sheard, S., Cook, S., Honour, E., Hybertson, D., Krupa, J., McEver, J., McKinney, D., Ondrus, P., Ryan, A., Scheurer, R. \& Singer, J. 2015. A complexity primer for systems engineers. INCOSE Complex Systems Working Group White Paper. Accessible via http://www.incose.org/docs/defaultsource/ProductsPublications/a-complexity-primer-for-systems-engineers.pdf [visited January 2020]

Sheehan J.H., P.H. Deitz, B.E. Bray, B.A. Harris, A.B. Wong. 2004. The Military Missions and Means Framework. Technical Report TR-756, Army Material Systems Analysis Activity Aberdeen Proving Ground, MD.

Slantchev, B. L. 2012. Game theory: preferences and expected utility; $2^{\text {nd }}$ Edition. Technical Report, Political Science Courses, University of California: San Diego, CA.

Tolk, A., P. F. Katina, A. Rainville, \& J. Selby. 2020. Towards a framework for enterprise-wide operational agility in complex environments. Accepted by Int. J. System of Systems Engineering, DOI: 10.1504/IJSSE.2020.10025774.

TRADOC Pamphlet TP525-3-1. The U.S Army in Multi-Domain Operations 2018, 19-20.

Velasquez, M. \& P. T. Hester. 2013. An analysis of multi-criteria decision making methods. International Journal of Operations Research, 10(2), 56-66.

\section{AUTHOR BIOGRAPHIES}

ANDREAS TOLK is Senior Principal Computer Scientist at The MITRE Corporation in Hampton, Virginia, and adjunct Full Professor at Old Dominion University in Norfolk, Virginia. He holds a PhD and MSc in Computer Science from the University of the Federal Armed Forces of Germany. He is a Fellow of the Society for Modeling and Simulation and senior member in IEEE and ACM. His ORCID is 0000-00024201-8757, his email address is atolk@mitre.org.

KEVIN T. COMER is Senior Simulation Modeling Engineer at The MITRE Corporation in McLean, Virginia, and an Adjunct Professor at George Mason University, Fairfax, Virginia. He holds a PhD in Computational Social Science and a MSc in Operations Research from George Mason University, and a BSc in Systems Engineering from the University of Virginia. His email is ktcomer@mitre.org.

KHUONG DINH is Lead Model-based Systems Engineer at The MITRE Corporation in Honolulu, Hawaii. He holds a BSc in Mathematics from Longwood College. He has long-standing experiences in support of defense forces with model-based and simulation methods. His email is kdinh@mitre.org.

STEPHEN L. SCOTT is Principal Multi-Disciplinary Systems Engineer at The MITRE Corporation in McLean, Virginia, and an Adjunct Professor at George Mason University, Fairfax, Virginia. He holds a $\mathrm{PhD}$ in Computational Social Science and a MSc in Computer Science from George Mason University. He published two books on agent-based applications. His email is slscott@mitre.org. 